\title{
SARS-CoV-2 Infection in Ivory Coast: A Serosurveillance Survey among Gold Mine Workers
}

\author{
Jean Marie Milleliri, ${ }^{1}$ Daouda Coulibaly, ${ }^{2}$ Blaise Nyobe, ${ }^{3}$ Jean-Loup Rey, ${ }^{1}$ Franck Lamontagne, ${ }^{1}$ Laurent Hocqueloux,${ }^{4}$ \\ Susanna Giaché, ${ }^{4}$ Antoine Valery, ${ }^{5}$ and Thierry Prazuck ${ }^{4 *}$ \\ ${ }^{1}$ Groupe d'intervention en sante Publique et Epidemiologie, Marseille, France; ${ }^{2}$ Institut National de sante Publique, Abidjan, Cote d'Ivoire; \\ ${ }^{3}$ Medicis, Urgences, Abidjan, Cote d'Ivoire; ${ }^{4}$ Maladies Infectieuses et Tropicales, Centre Hospitalier Regional d'Orleans, Orleans, France; \\ ${ }^{5}$ Departement d'Information Medicale, Centre Hospitalier Regional d'Orleans, Orleans, France
}

\begin{abstract}
Of the 107 million COVID-19 cases worldwide, less than 2 million have been reported in African countries. The aim of this study was to evaluate the seroprevalence of SARS-CoV-2 infection in Ivory Coast mine workers. From July 15 to October 13, 2020, a voluntary serological test campaign was conducted in 3 sites: two gold mines, and the headquarters in Abidjan. Rapid tests to detect IgG and IgM on capillary blood were performed. To identify independent sociodemographic characteristics associated with a higher SARS-CoV-2 seroprevalence rate, a multivariate logistic regression analysis was performed. A total of 1,687 subjects were tested; $91 \%$ were male $(n=1,536)$, and the mean age was 37 years. The overall seroprevalence was $25.1 \%(n=422)$, ranging between $13.6 \%(11.2-16.1 \%), 34.4 \%$ (31.1-37.7\%), and 34.7\% (26.2-43.2\%) in mine A, in mine B, and in Abidjan, respectively. Among the 422 seropositive subjects, 74 reported mild symptoms in the three previous months and one was hospitalized for severe COVID-19 infection. SARS-CoV-2 seroprevalence is high in both gold miners and administrative staff working in Ivory Coast. The burden of infection in West Africa has probably been underestimated till now.
\end{abstract}

COVID-19 represents an unprecedented international public health challenge responsible for significant morbidity and mortality. In March 2020, the WHO officially declared the COVID-19 pandemic. As of February 10, 2021, (SARS-CoV-2) caused 2,360,000 deaths of more than 107 million cases reported worldwide. Unfortunately, these numbers continue to increase every day. ${ }^{1}$

Officially, according to the latest February 7, $2021 \mathrm{WHO}$ report, COVID-19 cases in Africa were 2,761,000 and COVID19-related deaths were $65,736 .^{2}$

Considering the daily increase in the number of COVID-19 cases all around the world, it appears that Africa is controlling the epidemic spread better than Europe, the United States, or South America. Catastrophic scenarios about potentially devastating effect of COVID-19 in Africa have fortunately not yet occurred. The reasons are multiple and not always clearly established. ${ }^{3}$ Some authors argue that this epidemic reached Africa a few weeks after European and African leaders adopted preventive measures, thanks to acquired knowledge of what was happening in Europe, having probably learned important lessons from previous major outbreaks, like 2014 Ebola virus disease in West Africa. In addition, many parts of Africa are still isolated and not linked to significant tourism and international business-related traffic. However, according to recent data, SARS-CoV-2 serological positivity in South Africa appears to be so high that it approaches the herd immunity threshold (i.e., more than 60\%). ${ }^{4}$ Did SARS-CoV-2 infection largely and silently spread in Africa, or did factors such as environment, younger population, climate, and policies limit the penetration of the virus in most African countries? In this report, we present the results of a COVID-19 serological testing campaign conducted in Ivory Coast among employees of a gold mining company using a reliable rapid point-of-care antibody test.

The objective of this testing campaign was to sensitize and protect employees and their families, particularly when a recent SARS-CoV-2 infection was diagnosed.

*Address correspondence to Thierry Prazuck, Centre Hospitalier Regional d'Orleans, 14 Ave. de l'hopital Orleans, Cedex 2, Orleans 45067, France. E-mail: thierry.prazuck@chr-orleans.fr

\section{SUBJECTS AND METHODS}

The serological screening based on voluntary testing was conducted in the period between July 15 and October 13 in two mines and in the Abidjan headquarter mines $A$ and $B$, located, respectively, at $500 \mathrm{~km}$ and $200 \mathrm{~km}$ northwest from the economic capital of the country (Abidjan). Threethousand three hundred eighty people are currently working in these three sites, including the Abidjan headquarters. Of these 3,380 workers, 1,360 are direct employees and 2,020 are subcontractor's employees of different companies. Direct employees working in the mines live on site in a rather confined place, whereas the subcontractor's employees come back home in their villages/towns every day. Administrative staff and other workers located in Abidjan headquarters are mainly exposed to COVID-19 in their daily activities in contact with the Ivorian urban population of Abidjan.

All employees were informed about the objectives of the campaign and were invited to participate. No clinical criteria were required to be tested. Tests were performed at the different work sites by healthcare personnel according to the manufacturers' instructions after a 2-hour training session. For each participant, written informed consent was obtained. Test result was provided within 15 minutes. Each time a test is carried out, a data sheet is filled out including socio-professional characteristics, including age, gender, nationality, place of residence, and employment. Each completed form is entered into an Excel database, and photographs of positive assay results were saved.

A standardized questionnaire was filled for each subject, including age, gender, working status, and previous symptoms potentially related to a SARS-CoV-2 infection in the previous 5 months (i.e., fever, dyspnea, cough, flu-like syndrome, and anosmia/ageusia).

\section{POINT-OF-CARE USED TEST}

The COVID-PRESTO ${ }^{\circledR}$ SARS-CoV-2 lgG/lgM antibody test kit (AAZ LMB, Boulogne Billancourt, France) is a lateral flow 
immune-chromatographic assay targeting antibodies specific to the SARS-CoV-2 N-protein. It has been manufactured and marketed by AAZ-LMB and already approved by French authorities. This rapid diagnostic test (RDT) uses antihuman IgM antibodies (test line $\operatorname{lgM}$ ), antihuman IgG antibodies (test line $\operatorname{lgG}$ ), and rabbit $\lg$ (control line $C$ ) immobilized on a nitrocellulose strip. The conjugate (recombinant SARS-CoV-2 antigens labeled with colloidal gold) is also integrated into the strip. When a specimen is well placed in the sample and the assay buffer is added, the $\operatorname{lgM}$ and $\lg G$ antibodies, if present, bind to the SARS-CoV-2 conjugates, forming an antigenantibodies complex. This complex migrates through a nitrocellulose membrane by capillary action. When the complex meets the line of the corresponding immobilized antibodies (antihuman IgM and/or antihuman IgG), the complex is trapped and forms a burgundy-colored band confirming test result. Results must be read within 10 minutes by two independent operators. When the control line is the only one present, the sample is negative. If the control line does not appear, the test is invalid and should be repeated with a new cassette. The sensitivity of COVID-PRESTO test ranged from $10 \%$ for patients having experienced their first symptoms from 0 to 5 days earlier to $100 \%$ in patients whose symptoms occurred more than 15 days earlier. ${ }^{5}$

\section{STATISTICAL ANALYSIS}

Data were collected on an Excel spreadsheet. Data were categorized by mine site, gender, age, and working characteristics (i.e., direct employees versus subcontractor's employees). Qualitative and quantitative variables including the proportion of seropositivity were reported in percentage and a 95\% Cl. Multivariate logistic regression was used to determine the relationship between demographics characteristics and seropositivity to SARS-CoV-2. A P-value of less than 0.05 was regarded as statistically significant. Only statistically significant variables were introduced in the multivariate model. Analysis was carried out using $\mathrm{R}$ studio software.

\section{ETHICAL CONSIDERATIONS}

The GISPE Research and Ethical Committee approved the survey on May 15, 2020 (N EC-GISPE014). Potential participants were informed about the campaign purpose and procedures.

\section{RESULTS}

Among the 3,380 employees, a total of 1,687 subjects $(49.9 \%)$ were included in the study. Based on volunteering testing, only subjects who participate in the screening procedure were analyzed. One thousand five hundred thirty-five were males and 151 were females. The mean age was 37 (18-66) years; 1,566 employees (92.7\%) worked in the mine sites and 121 (7.3\%) in Abidjan. Among mining workers, 845 were direct employees living night and day in the compound (587 in mine A and 258 in mine B), and 718 were subcontractor's employees of other companies working in the mine during the day but coming back to village after work (213 in mine $A$ and 501 in mine B). One thousand four hundred fiftyeight workers were native from Ivory Coast (86.4\%), 143 from another African country (8.5\%), and the remaining 87 (5.1\%)
TABLE 1

Sociodemographic characteristics of the study population

\begin{tabular}{lccccr}
\hline \multicolumn{1}{c}{ Characteristics } & Overall & Ity & Agbaou & Abidjan & $P$-value \\
\hline$N$ & 1,687 & 803 & 763 & 121 & \\
Gender ratio (M/F) & 10.2 & 14.1 & 10.1 & 3.17 & $<0.001$ \\
Mean age (SD) (years) & $38(8.6)$ & $36(8.3)$ & $37(8.7)$ & $39(9.7)$ & 0.001 \\
$\quad<36$ & 805 & 400 & 359 & 46 & \\
$\quad 36-49$ & 733 & 338 & 336 & 59 & NS \\
$\geq 50$ & 149 & 65 & 68 & 16 & \\
Employee's status & & & & & \\
$\quad$ Direct employees & 848 & 587 & 258 & 3 & \\
$\quad$ Subcontractors & 721 & 213 & 505 & 3 & $<0.001$ \\
$\quad$ Administrative staff & 118 & 3 & & 115 & \\
\hline NS = not significant or $P>0.05$. & & & &
\end{tabular}

came from 12 worldwide countries (details in Table 1). Subjects working in Abidjan were significantly older (63\% older than 36 years), and the proportion of male workers was half compared with mine sites.

Among the 1,687 people included in the study, 422 had a positive serological test (either lgG and/or lgM). Overall, seroprevalence was $25.1 \%$, and prevalence rates were similar when stratified by age or gender. According to the different working sites, seroprevalence was $34.4 \%$, 34.7\%, and $13.6 \%$ in mine A, Abidjan headquarters, and mine B, respectively. The seroprevalence rate was significantly lower in subcontractors' workers than in those living full time in the mines. Ivory Coast natives had the highest prevalence rate (26.5\% [24.3-28.8\%]). Conversely, people coming from countries outside Africa were less likely to be SARS-CoV-2 seropositive (details in Table 2).

Among the 422 seropositive subjects, only one had previously been hospitalized for suspected COVID-19. Eighteen additional subjects had mild symptoms and were diagnosed by a positive real-time reverse transcription-PCR (RT-PCR) test. Among the 404 remaining subjects, 74 reported mild nonspecific symptoms that may or may not be attributable to a SARS-CoV-2 infection, particularly in a tropical setting, but were not tested at the time of symptoms.

No data are available on those who were not yet tested at the time of analysis. Some were in vacation, some were not volunteers, and some were not available for any reason on the days of the screening procedure. Then, it was not possible to make any comparison between those tested and those not tested.

\section{DISCUSSION}

The current epidemiological study conducted on a specific African population reports SARS-CoV-2 seroprevalence as high as $35 \%$ in gold mining workers as well as in administrative staff living in Abidjan. This high seroprevalence rate was reached before July 2020, and it remained stable after the first pandemic wave, between July and October 2020. This silent outbreak was not previously detected because most cases were asymptomatic.

From March to December 1, 2020, Ivory Coast reported 21,250 SARS-CoV-2 infections and 127 COVID-19-related deaths, suggesting that only $0.084 \%$ of Ivory Coast's 25 million inhabitants had been infected. ${ }^{2}$ Official available data do not reflect the burden of infection but may reflect the emerging part of the iceberg. To our knowledge, there are very few published studies evaluating SARS-CoV-2 seroprevalence in 
TABLE 2

SARS-CoV-2 seroprevalence rates, odds ratios, and $95 \% \mathrm{Cl}$ according to sociodemographic characteristics plus adjusted odds ratio (logistic model)

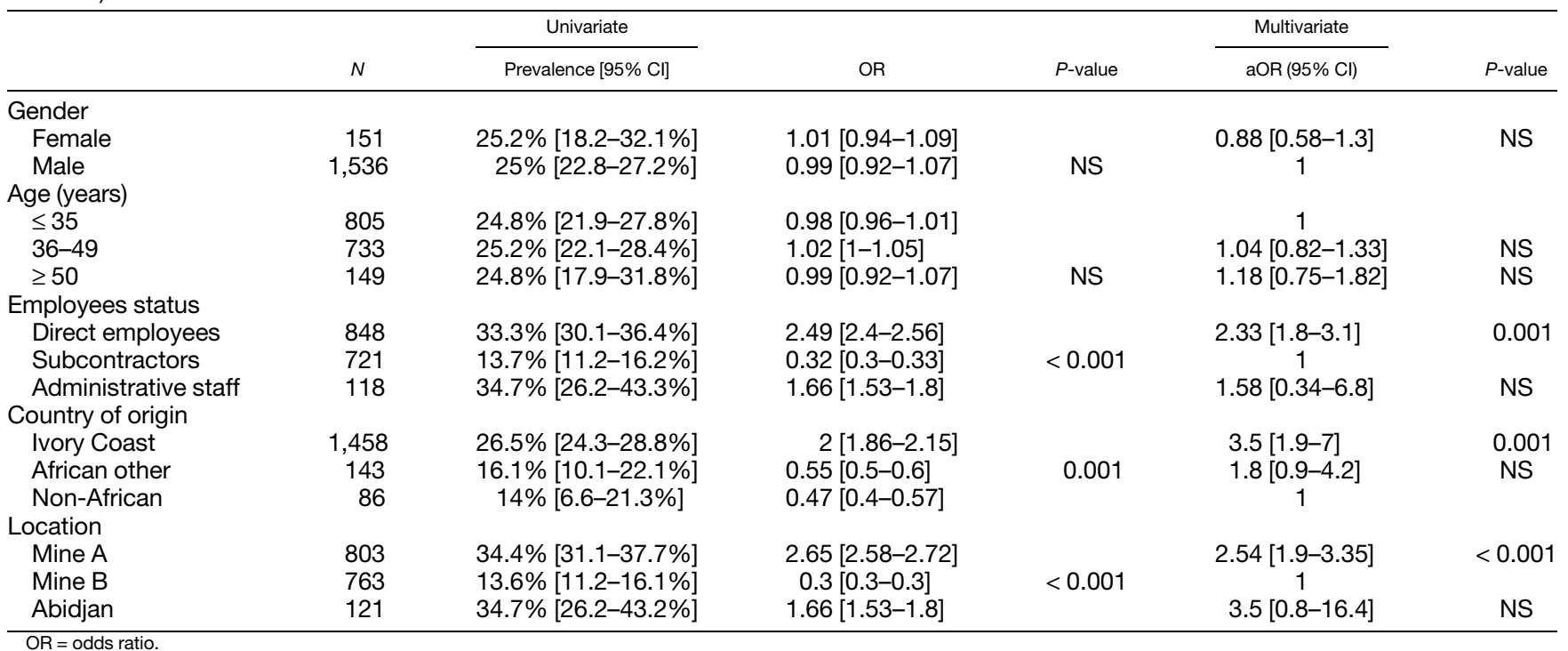

African countries. In Togo, among 955 participants from five different sectors, the prevalence rate was $0.9 \%(95 \% \mathrm{Cl}$ : $0.4-1.8 \%) .{ }^{6}$ In a study conducted in Kenya from April to June 2020 on 3,174 blood donors' samples, seroprevalence was $5.2 \%(95 \% \mathrm{Cl}: 3.7-7.1 \%){ }^{7}$ In two small populations of household contacts and healthcare workers in Nigeria, seroprevalence rates were to $25.4 \%$ and $45 \%$, respectively. ${ }^{8,9}$ Among the available preprint or published studies, seroprevalence was evaluated using RDTs in three studies, ELISA in three studies, and Clinical Laboratory Improvement Amendments in one study. ${ }^{6-12} A$ recent study aiming to detect the persistence of SARS-CoV-2 antibodies 3-4 months after the onset of symptoms in healthcare workers at the Strasbourg University hospital reported that ELISA serological test has a $59 \%$ sensitivity and Biosynex RDT a $85 \%$ sensitivity compared with the S-flow reference test developed by the French National Reference Center for SARS-CoV-2 infection (Pasteur Institute, Paris, France). ${ }^{13}$ The RDT used in our study showed high sensitivity even more than 21 days after the onset of symptoms, as well as a $100 \%$ specificity in a study carried out independently from the manufacturer. ${ }^{14}$ Hence, seroprevalence rates from studies using ELISA tests must be cautiously interpreted as they may underestimate the real data.

In our study, $14-35 \%$ of workers were SARS-CoV-2 seropositive, despite having no symptoms or at least no severe disease. These data appear to be quite different than those observed in the rest of the world, but similar results have already been described in Ibadan, Nigeria, in healthcare workers who are much more exposed to the infection than mine workers. ${ }^{9}$ A high level of seropositivity was found in both mining workers and administrative staff living in the economic capital. In mine A, as people live together, a cluster phenomenon may have occurred, explaining the difference in seroprevalence with mine $\mathrm{B}$, which was more confined during the first wave with limited circulation between mine $B$ and Abidjan. Subjects living in mine $B$ had the same life habits as the African population living in Abidjan. Therefore, we can expect that this similar seroprevalence reflects the real one in Abidjan.

In mine B, subcontractors' workers constitute $67 \%$ of the working population compared with $29 \%$ in mine A. As subcontractors' worker do not live full time in the mine, the lower seroprevalence of this population could reflect a lower prevalence in rural areas of the country.

In the capital, seropositivity was $34.7 \%$ among the 121 subjects working in the administrative staff in Abidjan and having no contact with gold mines. These data could reflect the high SARS-CoV-2 seroprevalence in the general population living in Abidjan.

Nevertheless, the most surprising results of the current study are the low percentage of symptomatic cases and mortality rate. Some authors speculate that African SARSCoV-2 seroprevalence data could be related to a younger population, a favorable climate, and possible preexisting immunity due to previous exposure to other coronaviruses. ${ }^{14}$ In our study, the mean age was 37 years, and there was no increase in symptom incidence or hospitalization according to age range. Lipsitch et al. ${ }^{15}$ explored four models of crossreactive immunity. Implications for individuals based on the reduction of lung burden model are a reduction in the magnitude and duration of symptomatic/clinical disease without change in the upper respiratory tract SARS-CoV-2 viral load. This model could explain low clinical expression despite high prevalence rates in the population studied.

Relatively stable prevalence rates observed between July and September may indicate that the SARS-CoV-2 outbreak occurred as early as March or April as in Europe, mainly not to say exclusively from imported cases, and spread silently but rapidly across the country. The plateau observed since July could be related either to complete travel restrictions, to the end of the first epidemic wave as in Europe, to the nationwide control of the SARS-CoV-2 circulation, or may reflect other phenomena including preexisting immunity, 
genetic factors, or other unexplained factors in the remaining population.

This study has some limitations. First, the included population is not representative of the general Ivoirian population, although subjects living in Abidjan have presumably similar habits to those of the Abidjan's population. Furthermore, the voluntary test may have selected people thinking to be more exposed to the virus, for example, people who previously had mild symptoms or had household contacts of a confirmed SARS-CoV-2 case. Being an operational protocol, screening was based on employee volunteering. There was therefore no refusal to be screened, but on the contrary, there was broad support. Third, even if COVID-PRESTO has an excellent diagnostic performance, diagnostic sensitivity decreases a few months after the infection, compared with the reference test S-Flow, and may therefore have underestimated the real seroprevalence.

In conclusion, the high SARS-CoV-2 prevalence rate among mining workers recruited for this study confirms a higher proportion of asymptomatic cases than that observed in Europe, South America, or the United States till now. Herd immunity could be achieved in Africa much easier than expected without generating a dramatic health crisis.

Received January 27, 2021. Accepted for publication February 25, 2021.

Published online March 18, 2021.

Acknowledgment: The American Society of Tropical Medicine and Hygiene has waived the Open Access fee for this article due to the ongoing COVID-19 pandemic.

Financial support: No funding J. M. M., Groupe d'intervention en sante publique et epidemiologie, Marseille, France, j-m.milleliri@wanadoo.fr.

Authors' addresses: Jean Marie Milleliri, Jean-Loup Rey, Franck Lamontagne, Groupe d'intervention en sante publique et epidemiologie, Marseille, France, E-mails: j-m.milleliri@wanadoo.fr, jeanloup.rey@wanadoo.fr, and flamontagne512@gmail.com. Daouda Coulibaly, Institut National de sante publique, Abidjan, Lagunes, Cote d'Ivoire, E-mail: daocoul@yahoo.fr. Blaise Nyobe, Medicis, Urgences, Abidjan, Cote d'Ivoire, E-mail: medcoordinator@medicis-ci.com. Laurent Hocqueloux, Susanna Giaché,Thierry Prazuck,Maladies infectieuses et tropicales, Centre Hospitalier Regional d'Orleans, Orleans, France, E-mails: laurent.hocqueloux@chr-orleans.fr, susanna.giache@chr-orleans.fr, and thierry.prazuck@chr-orleans.fr.
This is an open-access article distributed under the terms of the Creative Commons Attribution (CC-BY) License, which permits unrestricted use, distribution, and reproduction in any medium, provided the original author and source are credited.

\section{REFERENCES}

1. World Health Organization, 2021. WHO Coronavirus Disease (COVID-19) Dashboard. Available at: https://covid19.who.int/ table.

2. World Health Organization, 2021. COVID-19 Situation Update for the Weekly Epidemiological Report Update on 7 February 2021. Available at: https://www.who.int/publications/m/item/weeklyepidemiological-update---9-february-2021.

3. Payne C, 2020. COVID-19 in Africa. Nat Hum Behav 4: 436-437.

4. Myers J, 2020. MAVERICK CITIZEN OP-ED: Covid-19: After a severe epidemic, South Africa appears to be approaching some herd immunity. Daily Maverick. Disponible sur: https:// www.dailymaverick.co.za/article/2020-10-07-covid-19-after-asevere-epidemic-south-africa-appears-to-be-approachingsome-herd-immunity/.

5. Prazuck T et al., 2020. Evaluation of performance of two SARS-CoV2 Rapid IgM-IgG combined antibody tests on capillary whole blood samples from the fingertip. PLoS One 15: e0237694.

6. Halatoko WA et al., 2020. Prevalence of SARS-CoV-2 among high-risk populations in Lomé (Togo) in 2020. PLoS One 15: e0242124.

7. Uyoga $S$ et al., 2021. Seroprevalence of anti-SARS-CoV-2 IgG antibodies in Kenyan blood donors. Science 371: 79-82.

8. Asuquo Ml et al., 2021. Prevalence of IgG and IgM antibodies to SARS-CoV-2 among clinic staff and patients. medRxiv. doi: 2020.07.02.20145441.

9. Olayanju O et al., 2020. SARS-CoV-2 seropositivity in asymptomatic frontline health workers in Ibadan, Nigeria. Am J Trop Med Hyg104: 91-94.

10. Majiya $\mathrm{H}$ et al., 2020. Seroprevalence of COVID-19 in Niger state. medRxiv. doi: 2020.08.04.20168112.

11. Kempen JH et al., 2020. SARS-CoV-2 serosurvey in Addis Ababa, Ethiopia. Am J Trop Med Hyg 103: 2022-2023.

12. Chibwana MG et al., 2020. High SARS-CoV-2 seroprevalence in health care workers but relatively low numbers of deaths in urban Malawi. medRxiv. doi: 10.1101/2020.07.30.20164970.

13. Grzelak $L$ et al., 2020. Sex differences in the decline of neutralizing antibodies to SARS-CoV-2. medrxiv doi: 2020.11.12.20230466.

14. Njenga MK et al., 2020. Why is there low morbidity and mortality of COVID-19 in Africa? Am J Trop Med Hyg 103: 564-569.

15. Lipsitch M, Grad YH, Sette A, Crotty S, 2020. Cross-reactive memory $\mathrm{T}$ cells and herd immunity to SARS-CoV-2. Nat Rev Immunol 20: 709-713. 